PROCEEDINGS OF THE

AMERICAN MATHEMATICAL SOCIETY

Volume 139, Number 7, July 2011, Pages 2399-2406

S 0002-9939(2010)10710-4

Article electronically published on December 16, 2010

\title{
THE POSITIVITY OF THE FIRST COEFFICIENTS OF NORMAL HILBERT POLYNOMIALS
}

\author{
SHIRO GOTO, JOOYOUN HONG, AND MOUSUMI MANDAL
}

(Communicated by Irena Peeva)

\begin{abstract}
Let $R$ be an analytically unramified local ring with maximal ideal $\mathfrak{m}$ and $d=\operatorname{dim} R>0$. If $R$ is unmixed, then $\overline{\mathrm{e}}_{I}^{1}(R) \geq 0$ for every $\mathfrak{m}$-primary ideal $I$ in $R$, where $\overline{\mathrm{e}}_{I}^{1}(R)$ denotes the first coefficient of the normal Hilbert polynomial of $R$ with respect to $I$. Thus the positivity conjecture on $\overline{\mathrm{e}}_{I}^{1}(R)$ posed by Wolmer V. Vasconcelos is settled affirmatively.
\end{abstract}

\section{INTRODUCTION}

Throughout this paper let $R$ be a Noetherian local ring with maximal ideal $\mathfrak{m}$ and $d=\operatorname{dim} R>0$. Assume that $R$ is analytically unramified, whence the $\mathfrak{m}$-adic completion $\widehat{R}$ of $R$ is reduced. We fix an $\mathfrak{m}$-primary ideal $I$ in $R$ and denote by $\overline{I^{n+1}}\left(\operatorname{resp} . \lambda_{R}\left(R / \overline{I^{n+1}}\right)\right)$ the integral closure of $I^{n+1}$ (resp. the length of $R / \overline{I^{n+1}}$ ) for each $n \geq 0$. Then the normal Hilbert function

$$
\lambda_{R}\left(R / \overline{I^{n+1}}\right)
$$

of $R$ with respect to $I$ is of polynomial type with degree $d$, and we have integers $\left\{\overline{\mathrm{e}}_{I}^{i}(R)\right\}_{0 \leq i \leq d}$ such that the equality

$$
\lambda_{R}\left(R / \overline{I^{n+1}}\right)=\overline{\mathrm{e}}_{I}^{0}(R)\left(\begin{array}{c}
n+d \\
d
\end{array}\right)-\overline{\mathrm{e}}_{I}^{1}(R)\left(\begin{array}{c}
n+d-1 \\
d-1
\end{array}\right)+\cdots+(-1)^{d} \overline{\mathrm{e}}_{I}^{d}(R)
$$

holds true for all $n \gg 0$. We call these integers $\overline{\mathrm{e}}_{I}^{i}(R)$ the coefficients of the normal Hilbert polynomial of $R$ with respect to $I$.

In this paper we are interested in the analysis of the first coefficient $\overline{\mathrm{e}}_{I}^{1}(R)$ of the normal Hilbert polynomial. The main purpose is to study the positivity conjecture on $\overline{\mathrm{e}}_{I}^{1}(R)$ posed by Wolmer V. Vasconcelos $[\mathrm{V}$, and our result is stated as follows.

Theorem 1.1. Let $R$ be an analytically unramified local ring with maximal ideal $\mathfrak{m}$ and $d=\operatorname{dim} R>0$. If $R$ is unmixed, then

$$
\overline{\mathrm{e}}_{I}^{1}(R) \geq 0
$$

for every $\mathfrak{m}$-primary ideal I in $R$.

Received by the editors July 1, 2010 .

2010 Mathematics Subject Classification. Primary 13H10; Secondary 13A30, 13B22, 13H15.

Key words and phrases. Associated graded ring, Rees algebra, normal ideal, normal Hilbert polynomial.

(C)2010 American Mathematical Society Reverts to public domain 28 years from publication 
Here we should note that the conjecture holds true in the case where $R$ is a Cohen-Macaulay local ring ([PUV, Theorem 2.2]). In fact, generally we have

$$
\overline{\mathrm{e}}_{I}^{0}(R)=\mathrm{e}_{I}^{0}(R),
$$

where $\mathrm{e}_{I}^{0}(R)$ stands for the ordinary Hilbert-Samuel multiplicity of $R$ with respect to $I$. Therefore $\overline{\mathrm{e}}_{I}^{1}(R) \geq \mathrm{e}_{I}^{1}(R)$, and so, if $R$ is a Cohen-Macaulay local ring, we get

$$
\overline{\mathrm{e}}_{I}^{1}(R) \geq \mathrm{e}_{I}^{1}(R) \geq 0,
$$

because $\mathrm{e}_{I}^{1}(R) \geq 0$ (N, Corollary 1]). Mainly based on this fact, the third author, M. Mandal, along with B. Singh and J. Verma [MSV], gave several interesting answers in certain special cases, and our Theorem 1.1 now affirmatively settles the conjecture in full generality.

We shall prove Theorem 1.1 in Section 2. In Section 3 we will discuss a few results related to the positivity conjecture. We expect that the integral closure $\bar{R}$ of $R$ is a regular ring and $I \bar{R}$ is normal; that is, $I^{n} \bar{R}$ is integrally closed for all $n \geq 1$, once $\overline{\mathrm{e}}_{I}^{1}(R)=0$ for some $\mathfrak{m}$-primary ideal $I$ in $R$. We shall give an affirmative answer in the case where $\bar{R}$ is a Cohen-Macaulay ring.

Throughout this paper, unless otherwise specified, we denote by $R$ a Noetherian local ring with maximal ideal $\mathfrak{m}$ and $d=\operatorname{dim} R>0$. Let $\bar{R}$ be the integral closure of $R$ in its total quotient ring. For each finitely generated $R$-module $M$, let $\mu_{R}(M)$ (resp. $\lambda_{R}(M)$ ) stand for the number of elements in a minimal system of generators (resp. the length) of $M$.

\section{Proof of Theorem 1.1}

The main purpose of this section is to prove Theorem 1.1

Proof of Theorem 1.1. We have $\overline{\mathrm{e}}_{I \widehat{R}}^{1}(\widehat{R})=\overline{\mathrm{e}}_{I}^{1}(R)$, since $\overline{\mathfrak{a} \widehat{R}}=\overline{\mathfrak{a}} \widehat{R}$ for every $\mathfrak{m}$ primary ideal $\mathfrak{a}$ in $R$. Therefore, passing to the $\mathfrak{m}$-adic completion $\widehat{R}$ of $R$, without loss of generality we may assume that $R$ is complete. If $d=1$, we then have

$$
\overline{\mathrm{e}}_{I}^{1}(R)=\lambda_{R}(\bar{R} / R) \geq 0 .
$$

Suppose that $d \geq 2$ and let $S=\bar{R}$. For each $\mathfrak{p} \in \operatorname{Ass} R$ we put $S(\mathfrak{p})=\overline{R / \mathfrak{p}}$. Then $S(\mathfrak{p})$ is a module-finite extension of $R / \mathfrak{p}$, and we get

$$
S=\prod_{\mathfrak{p} \in \operatorname{Ass} R} S(\mathfrak{p}) \text { and } \overline{I^{n+1}}=\overline{I^{n+1} S} \cap R
$$

for all $n \geq 0$. Hence

$$
\begin{aligned}
\lambda_{R}\left(R / \overline{I^{n+1}}\right) \leq \lambda_{R}\left(S / \overline{I^{n+1} S}\right) & =\sum_{\mathfrak{p} \in \operatorname{Ass} R} \lambda_{R}\left(S(\mathfrak{p}) / \overline{I^{n+1} S(\mathfrak{p})}\right) \\
& =\sum_{\mathfrak{p} \in \operatorname{Ass} R} \lambda_{R}\left(S(\mathfrak{p}) / \mathfrak{m}_{S(\mathfrak{p})}\right) \cdot \lambda_{S(\mathfrak{p})}\left(S(\mathfrak{p}) / \overline{I^{n+1} S(\mathfrak{p})}\right),
\end{aligned}
$$


where $\mathfrak{m}_{S(\mathfrak{p})}$ denotes the maximal ideal of $S(\mathfrak{p})$. Notice that, since $\operatorname{dim} S(\mathfrak{p})=d$ for each $\mathfrak{p} \in$ Ass $R$, we have

$$
\begin{aligned}
\overline{\mathrm{e}}_{I}^{0}(R)=\mathrm{e}_{I}^{0}(R)=\mathrm{e}_{I}^{0}(S) & =\sum_{\mathfrak{p} \in \operatorname{Ass} R} \mathrm{e}_{I}^{0}(S(\mathfrak{p})) \\
& =\sum_{\mathfrak{p} \in \operatorname{Ass} R} \lambda_{R}\left(S(\mathfrak{p}) / \mathfrak{m}_{S(\mathfrak{p})}\right) \cdot \mathrm{e}_{I S(\mathfrak{p})}^{0}(S(\mathfrak{p})) \\
& =\sum_{\mathfrak{p} \in \operatorname{Ass} R} \lambda_{R}\left(S(\mathfrak{p}) / \mathfrak{m}_{S(\mathfrak{p})}\right) \cdot \overline{\mathrm{e}}_{I S(\mathfrak{p})}^{0}(S(\mathfrak{p})),
\end{aligned}
$$

whence

$$
\begin{aligned}
0 & \leq \lambda_{R}\left(S / \overline{I^{n+1} S}\right)-\lambda_{R}\left(R / \overline{I^{n+1}}\right) \\
& =\left[\overline{\mathrm{e}}_{I}^{1}(R)-\sum_{\mathfrak{p} \in \operatorname{Ass} R} \lambda_{R}\left(S(\mathfrak{p}) / \mathfrak{m}_{S(\mathfrak{p})}\right) \cdot \overline{\mathrm{e}}_{I S(\mathfrak{p})}^{1}(S(\mathfrak{p}))\right]\left(\begin{array}{c}
n+d-1 \\
d-1
\end{array}\right) \\
& +\quad \text { (terms of lower degree) },
\end{aligned}
$$

so that

$$
\overline{\mathrm{e}}_{I}^{1}(R) \geq \sum_{\mathfrak{p} \in \operatorname{Ass} R} \lambda_{R}\left(S(\mathfrak{p}) / \mathfrak{m}_{S(\mathfrak{p})}\right) \cdot \overline{\mathrm{e}}_{I S(\mathfrak{p})}^{1}(S(\mathfrak{p}))
$$

Thus, in order to see $\overline{\mathrm{e}}_{I}^{1}(R) \geq 0$, it suffices to show that $\overline{\mathrm{e}}_{I S(\mathfrak{p})}^{1}(S(\mathfrak{p})) \geq 0$ for each $\mathfrak{p} \in \operatorname{Ass} R$. If $d=2$, we get

$$
\overline{\mathrm{e}}_{I S(\mathfrak{p})}^{1}(S(\mathfrak{p})) \geq \mathrm{e}_{I S(\mathfrak{p})}^{1}(S(\mathfrak{p})) \geq 0,
$$

because $S(\mathfrak{p})$ is a Cohen-Macaulay local ring. Hence $\overline{\mathrm{e}}_{I}^{1}(R) \geq 0$.

Suppose that $d \geq 3$ and that our assertion holds true for $d-1$. Then thanks to the above observation, passing to the ring $S(\mathfrak{p})$, we may assume that $R$ is a normal complete local ring. Let $I=\left(a_{1}, a_{2}, \ldots, a_{\ell}\right)$ with $a_{i} \in R$, where $\ell=\mu_{R}(I)$. Let

$$
T=R\left[Z_{1}, Z_{2}, \ldots, Z_{\ell}\right], \quad \mathfrak{q}=\mathfrak{m} T, \quad x=\sum_{i=1}^{\ell} a_{i} Z_{i}, \quad \text { and } D=T / x T,
$$

where $Z_{1}, Z_{2}, \ldots, Z_{\ell}$ are indeterminates over $R$. Let

$$
R^{\prime}=T_{\mathfrak{q}}, \quad I^{\prime}=I R^{\prime}, \quad \text { and } D^{\prime}=D_{\mathfrak{q}} .
$$

We then have $\overline{I^{n+1} R^{\prime}}=\overline{I^{n+1}} R^{\prime}$ for all $n \geq 0$, so that $\lambda_{R^{\prime}}\left(R^{\prime} / \overline{I^{n+1} R^{\prime}}\right)=\lambda_{R}\left(R / \overline{I^{n+1}}\right)$, whence

$$
\overline{\mathrm{e}}_{I}^{1}(R)=\overline{\mathrm{e}}_{I^{\prime}}^{1}\left(R^{\prime}\right) .
$$

Here we notice that $\operatorname{Ass} D^{\prime}=\operatorname{Assh} D^{\prime}$, because $R^{\prime}$ is catenary and normal; hence $D^{\prime}$ is unmixed, as $D^{\prime}$ is a homomorphic image of a Cohen-Macaulay ring. The ring $D^{\prime}$ is analytically unramified. To see this, since $D^{\prime}$ is a Nagata local ring, by [M], Theorem 70] it suffices to show that $D$ is reduced; that is, $D_{P}=T_{P} / x T_{P}$ is an integral domain for every $P \in \operatorname{Ass}_{T} D$. Let $\mathfrak{p}=P \cap R$. Then since $\mathrm{ht}_{T} P=1$, we have $\mathrm{ht}_{R} \mathfrak{p} \leq 1$, so that $I \nsubseteq \mathfrak{p}$, because $\mathrm{ht}_{R} \mathfrak{p} \leq 1<d=\operatorname{dim} R$. Without loss of generality we may assume that $a_{\ell} \notin \mathfrak{p}$. Then, because $x=\sum_{i=1}^{\ell} a_{i} Z_{i}$ and $a_{\ell}$ is a unit of $R_{\mathfrak{p}}$, we get

$$
T_{\mathfrak{p}}=R_{\mathfrak{p}}\left[Z_{1}, Z_{2}, \ldots, Z_{\ell}\right]=R_{\mathfrak{p}}\left[Z_{1}, Z_{2}, \ldots, Z_{\ell-1}, x\right],
$$


whence the ring

$$
T_{\mathfrak{p}} / x T_{\mathfrak{p}}=R_{\mathfrak{p}}\left[Z_{1}, Z_{2}, \ldots, Z_{\ell}\right] / x R_{\mathfrak{p}}\left[Z_{1}, Z_{2}, \ldots, Z_{\ell}\right]=R_{\mathfrak{p}}\left[Z_{1}, Z_{2}, \ldots, Z_{\ell-1}\right]
$$

is an integral domain, as it is the polynomial ring with $\ell-1$ indeterminates over $R_{\mathfrak{p}}$. Therefore for all $P \in \operatorname{Ass}_{T} D$ the ring $D_{P}=T_{P} / x T_{P}$ is an integral domain, because it is a localization of $R_{\mathfrak{p}}\left[Z_{1}, Z_{2}, \ldots, Z_{\ell-1}\right]$. Thus $D$ is reduced, whence $D^{\prime}$ is analytically unramified and unmixed.

Let us denote by $\mathcal{A}$ the extended Rees ring of $I T$ and by $\overline{\mathcal{A}}$ the integral closure of $\mathcal{A}$ in $T\left[t, t^{-1}\right]$, where $t$ denotes an indeterminate. Similarly, let us denote by $\mathrm{T}$ the extended Rees ring of $I D$ and by $\overline{\mathrm{T}}$ the integral closure of $\mathrm{T}$ in $D\left[t, t^{-1}\right]$. We put $N=\left(t^{-1}, I t\right)$ in $\mathcal{A}$. We look at the homomorphism

$$
\psi: T\left[t, t^{-1}\right] \rightarrow D\left[t, t^{-1}\right]
$$

of graded $T$-algebras such that $\psi(t)=t$. Since $\psi(\mathcal{A})=\mathrm{T}$ and $\overline{\mathrm{T}}$ is a module-finite extension of $\mathrm{T}$, the homomorphism $\psi$ gives rise to the finite homomorphism

$$
\varphi: \overline{\mathcal{A}} / x t \overline{\mathcal{A}} \longrightarrow \overline{\mathrm{T}}
$$

of graded $T$-algebras. Let $\overline{\mathcal{B}}$ (resp. $\overline{\mathrm{U}}$ ) denote the integral closure of $\mathcal{B}=\mathcal{A}_{\mathfrak{q}}$ (resp. $\mathrm{U}=\mathrm{T}_{\mathfrak{q}}$ ). Then we get the homomorphism

$$
\varphi_{\mathfrak{q}}: \overline{\mathcal{B}} / x t \overline{\mathcal{B}} \rightarrow \overline{\mathrm{U}}
$$

of graded $R^{\prime}$-algebras and, thanks to the proof of [HU, Theorem 2.1], we furthermore have the following. Let us include a brief proof for the sake of completeness.

Claim 1. The homomorphism

$$
\varphi_{P}:[\overline{\mathcal{A}} / x t \overline{\mathcal{A}}]_{P} \longrightarrow[\overline{\mathrm{T}}]_{P}
$$

is an isomorphism for all $P \in \operatorname{Spec} \mathcal{A} \backslash V(N)$. Hence the kernel and the cokernel of the homomorphism $\varphi_{\mathfrak{q}}: \overline{\mathcal{B}} / x t \overline{\mathcal{B}} \longrightarrow \overline{\mathrm{U}}$ of graded $\mathcal{B}$-modules are of finite length, so they are finitely graded.

Proof. Because $\overline{\mathcal{A}}[t]=T\left[t, t^{-1}\right]$ and $x t \overline{\mathcal{A}}[t]=x T\left[t, t^{-1}\right]$, the homomorphism $\varphi_{t^{-1}}$ is an isomorphism, whence so is the homomorphism $\varphi_{P}$, if $t^{-1} \notin P$.

Suppose now that It $\nsubseteq P$. We may assume $a_{\ell} t \notin P$. Notice that

$$
\begin{aligned}
{[\overline{\mathcal{A}} / x t \overline{\mathcal{A}}]_{a_{\ell} t} } & =\left[\overline{R\left[I t, t^{-1}\right]}\left[Z_{1}, Z_{2}, \ldots, Z_{\ell}\right] / x t \cdot \overline{R\left[I t, t^{-1}\right]}\left[Z_{1}, Z_{2}, \ldots, Z_{\ell}\right]\right]_{a_{\ell} t} \\
& =\left(\overline{R\left[I t, t^{-1}\right]}\left[\frac{1}{a_{\ell} t}\right]\right)\left[Z_{1}, Z_{2}, \ldots, Z_{\ell}\right] /\left(\sum_{i=1}^{\ell-1} \frac{a_{i} Z_{i} t}{a_{\ell} t}+Z_{\ell}\right) \\
& =\left(\overline{R\left[I t, t^{-1}\right]}\left[\frac{1}{a_{\ell} t}\right]\right)\left[Z_{1}, Z_{2}, \ldots, Z_{\ell-1}\right]
\end{aligned}
$$


and that

$$
\begin{aligned}
D\left[t, t^{-1}\right]_{a_{\ell} t} & =T\left[t, t^{-1}, \frac{1}{a_{\ell} t}\right] / x \cdot T\left[t, t^{-1}, \frac{1}{a_{\ell} t}\right] \\
& =T\left[t, t^{-1}, \frac{1}{a_{\ell}}\right] / x \cdot T\left[t, t^{-1}, \frac{1}{a_{\ell}}\right] \\
& =R\left[\frac{1}{a_{\ell}}, Z_{1}, Z_{2}, \ldots, Z_{\ell}, t, t^{-1}\right] / x \cdot R\left[\frac{1}{a_{\ell}}, Z_{1}, Z_{2}, \ldots, Z_{\ell}, t, t^{-1}\right] \\
& =\left(R\left[\frac{1}{a_{\ell}}, t, t^{-1}\right]\right)\left[Z_{1}, Z_{2}, \ldots, Z_{\ell}\right] /\left(\sum_{i=1}^{\ell-1} \frac{a_{i} Z_{i}}{a_{\ell}}+Z_{\ell}\right) \\
& =\left(\left[R\left[t, t^{-1}\right]\right]\left[\frac{1}{a_{\ell} t}\right]\right)\left[Z_{1}, Z_{2}, \ldots, Z_{\ell-1}\right] .
\end{aligned}
$$

Then we get the following commutative diagram:

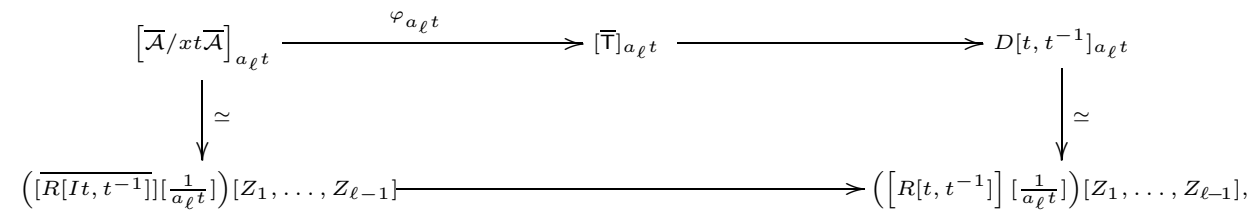

where the vertical homomorphisms are isomorphisms, so the horizontal homomorphism $\varphi_{a_{\ell^{t}}}$ is injective. Because $\left(\left[\overline{R\left[I t, t^{-1}\right]}\right]\left[\frac{1}{a_{\ell} t}\right]\right)\left[Z_{1}, Z_{2}, \ldots, Z_{\ell-1}\right]$ is integrally closed in $\left(\left[R\left[t, t^{-1}\right]\right]\left[\frac{1}{a_{\ell} t}\right]\right)\left[Z_{1}, Z_{2}, \ldots, Z_{\ell-1}\right]$ and $\varphi_{a_{\ell} t}$ is finite, $\varphi_{a_{\ell} t}$ is an isomorphism, whence

$$
\varphi_{P}:[\overline{\mathcal{A}} / x t \overline{\mathcal{A}}]_{P} \longrightarrow[\overline{\mathrm{T}}]_{P}
$$

is an isomorphism, too. This proves Claim 1

The normal ring $\overline{\mathcal{B}}$ is catenary, since it is a finitely generated $R^{\prime}$-algebra, while we get

$$
\operatorname{dim} \overline{\mathcal{B}} /\left(x t, t^{-1}\right) \overline{\mathcal{B}}=\operatorname{dim} \overline{\mathrm{U}} / t^{-1} \overline{\mathrm{U}}=d-1
$$

by Claim 1 . Therefore $t^{-1}, x t$ forms a regular sequence in the normal ring $\overline{\mathcal{B}}$. Hence $x t$ is a non-zerodivisor in the associated graded ring

$$
\overline{\mathcal{B}} / t^{-1} \overline{\mathcal{B}}=\bigoplus_{n \geq 0} \overline{I^{n}} R^{\prime} / \overline{I^{n+1}} R^{\prime}
$$

of the filtration $\left\{\overline{I^{n}} R^{\prime}\right\}_{n \in Z}$ of integrally closed ideals in $R^{\prime}$. Consequently, we have

$$
\overline{\mathrm{e}}_{I}^{1}(R)=\overline{\mathrm{e}}_{I^{\prime}}^{1}\left(R^{\prime}\right)=\overline{\mathrm{e}}_{I D^{\prime}}^{1}\left(D^{\prime}\right),
$$

since $\operatorname{dim} D^{\prime}=\operatorname{dim} R^{\prime}-1=d-1 \geq 2$ and since the kernel and the cokernel of the homomorphism

$$
\bar{\varphi}_{\mathfrak{q}}: \overline{\mathcal{B}} /\left(x t, t^{-1}\right) \overline{\mathcal{B}} \longrightarrow \overline{\mathrm{U}} / t^{-1} \overline{\mathrm{U}}
$$

induced from $\varphi_{\mathfrak{q}}$ are finitely graded. Thus the hypothesis of induction on $d$ yields the assertion that $\overline{\mathrm{e}}_{I}^{1}(R) \geq 0$, which completes the proof of Theorem 1.1.

The condition in Theorem 1.1 that $R$ is unmixed is not superfluous. Let us note the simplest example. See MSV, Example 2.4] for more examples. 
Example 2.1. We look at the local ring

$$
R=k[[X, Y, Z]] / \mathfrak{a},
$$

where $k[[X, Y, Z]]$ is the formal power series ring over a field $k$ and $\mathfrak{a}=(X) \cap(Y, Z)$. Then $\operatorname{dim} R=2, R$ is mixed, and $\bar{e}_{\mathfrak{m}}^{1}(R)=\bar{e}_{\mathfrak{m}}^{2}(R)=-1$. Hence the famous bad example [N, p. 203, Example 2] of Nagata which is a non-regular local integral domain $(A, \mathfrak{n})$ of dimension 2 with $\mathrm{e}_{\mathfrak{n}}^{0}(A)=1$ possess $\overline{\mathrm{e}}_{\mathfrak{n}}^{1}(A)=\overline{\mathrm{e}}_{\mathfrak{n}}^{2}(A)=-1$, because

$$
\widehat{A} \cong k[[X, Y, Z]] /[(X) \cap(Y, Z)]
$$

for some field $k$.

Proof. We put $T=k[[X, Y, Z]]$ and $\mathfrak{q}=(X, Y, Z)$ in $T$. Then $\bar{R}=T /(X) \oplus$ $T /(Y, Z)$, and we have the exact sequence

$$
0 \rightarrow R \rightarrow T /(X) \oplus T /(Y, Z) \rightarrow T / \mathfrak{q} \rightarrow 0
$$

of $T$-modules; hence $\mathfrak{m} \bar{R} \subseteq R$. Recall that $\mathfrak{m}$ is a normal ideal in $R$; that is, $\overline{\mathfrak{m}^{n}}=\mathfrak{m}^{n}$ for all $n \geq 1$, since the associated graded ring

$$
\operatorname{gr}_{\mathfrak{m}}(R)=k[X, Y, Z] /[(X) \cap(Y, Z)]
$$

of $\mathfrak{m}$ is reduced. Therefore, as

$$
\mathfrak{m}^{n+1}=\overline{\mathfrak{m}^{n+1}}=\overline{\mathfrak{m}^{n+1} \bar{R}} \cap R=\mathfrak{m}^{n+1} \bar{R} \cap R,
$$

thanks to exact sequence (E) above, we get

$$
0 \rightarrow R / \overline{\mathfrak{m}^{n+1}} \rightarrow T /\left[(X)+\mathfrak{q}^{n+1}\right] \oplus T /\left[(Y, Z)+\mathfrak{q}^{n+1}\right] \rightarrow T / \mathfrak{q} \rightarrow 0
$$

for all $n \geq 0$. Hence

$$
\lambda_{R}\left(R / \overline{\mathfrak{m}^{n+1}}\right)=\left(\begin{array}{c}
n+2 \\
2
\end{array}\right)+\left(\begin{array}{c}
n+1 \\
1
\end{array}\right)-1,
$$

so that $\overline{\mathrm{e}}_{\mathfrak{m}}^{1}(R)=\overline{\mathrm{e}}_{\mathfrak{m}}^{2}(R)=-1$.

Let us note a consequence of Theorem 1.1

Corollary 2.2 ([MTV, Theorem 1]). Let $R$ be an analytically unramified unmixed local ring with maximal ideal $\mathfrak{m}$ and $d=\operatorname{dim} R>0$. Let $I$ be a parameter ideal in $R$. If $\overline{\mathrm{e}}_{I}^{1}(R)=\mathrm{e}_{I}^{1}(R)$, then $R$ is a regular local ring with $\mu_{R}(\mathfrak{m} / I) \leq 1$, whence $I$ is normal.

Proof. We get $\mathrm{e}_{I}^{1}(R) \geq 0$ by Theorem 1.1, whence by [GhGHOPV, Theorem 2.1] $R$ is a Cohen-Macaulay local ring with $\mathrm{e}_{I}^{1}(R)=0$. Because $\overline{\mathrm{e}}_{\bar{I}}^{1}(R) \geq \mathrm{e}_{\bar{I}}^{1}(R)$ and

$$
\mathrm{e}_{\bar{I}}(R) \geq 0
$$

([Nr, Corollary 1]), we furthermore have $\mathrm{e}_{\bar{I}} \frac{1}{\mathrm{~N}}(R)=0$, whence $\bar{I}$ is a parameter ideal in $R$ ([Nr, Corollary 2]). Because parameter ideals contain no proper reductions ([NR]), we get $\bar{I}=I$, whence by [G, Theorem (3.1)] $R$ is a regular local ring with $\mu_{R}(\mathfrak{m} / I) \leq 1$ and $I$ is normal.

Remark 2.3. In Corollary 2.2, unless $I$ is a parameter ideal, $R$ is not necessarily a regular local ring, even though $\mathrm{e}_{I}^{1}(R)=\mathrm{e}_{I}^{1}(R)$. Let us note an example. We look at the local ring

$$
R=k[[X, Y, Z]] /\left(Z^{2}-X Y\right),
$$


where $k[[X, Y, Z]]$ is the formal power series ring over a field $k$ of characteristic 0 . Then $R$ is a rational singularity, so $\overline{\mathrm{e}}_{I}^{1}(R)=\mathrm{e}_{I}^{1}(R)$ for every integrally closed $\mathfrak{m}$-primary ideal $I$ in $R$.

\section{A further PRoblem}

Let $R$ be an analytically unramified unmixed local ring and $I$ an $\mathfrak{m}$-primary ideal in $R$. We then expect that $\bar{R}$ is a regular ring and $I \bar{R}$ is normal; that is, all the powers $I^{n} \bar{R}$ are integrally closed, once $\overline{\mathrm{e}}_{I}^{1}(R)=0$. This is the case when $\bar{R}$ is a Cohen-Macaulay ring, as we will show in the following.

Theorem 3.1. Let $R$ be an analytically unramified local ring with maximal ideal $\mathfrak{m}$ and $d=\operatorname{dim} R>0$. Let $S$ be an overring of $R$ and assume that $S$ is a finitely generated $R$-module with $\operatorname{dim}_{R} S / R<d$. Let $I$ be an $\mathfrak{m}$-primary ideal in $R$ such that $\overline{\mathrm{e}}_{I}^{1}(R)=0$. If $\operatorname{depth}_{R} S=d$, then $S$ is a regular ring, $S=\bar{R}$, and $I \bar{R}$ is normal.

Proof. We may assume that $R$ is complete. Let $\mathrm{Q}(R)$ be the total quotient ring of $R$. We notice that $S$ is a Cohen-Macaulay $R$-module with $\operatorname{dim}_{R} S=d$; hence $R$ is unmixed. Therefore $S \subseteq \mathrm{Q}(R)$, as $\operatorname{dim}_{R} S / R<d$, so that $S \subseteq \bar{R}$. Since $R$ is complete, we get a decomposition $S=\prod_{i=1}^{\ell} S_{i}$ of $S$, where $S_{i}$ is a Cohen-Macaulay local ring with $\operatorname{dim} S_{i}=d$. Consequently, for the same reason as in the proof of Theorem 1.1 we have

$$
\overline{\mathrm{e}}_{I}^{1}(R) \geq \sum_{i=1}^{\ell} \lambda_{R}\left(S_{i} / \mathfrak{m}_{i}\right) \cdot \overline{\mathrm{e}}_{I S_{i}}^{1}\left(S_{i}\right) \geq 0,
$$

where $\mathfrak{m}_{i}$ is the maximal ideal in $S_{i}$; hence $\overline{\mathrm{e}}_{I S_{i}}^{1}\left(S_{i}\right)=0$ for each $1 \leq i \leq \ell$. As $\overline{\mathrm{e}}_{I S_{i}}^{1}\left(S_{i}\right) \geq \mathrm{e} \frac{1}{I S_{i}}\left(S_{i}\right) \geq 0$, we have $\mathrm{e} \frac{1}{I S_{i}}\left(S_{i}\right)=0$, so that $\overline{I S_{i}}$ is a parameter ideal in $S_{i}$. Hence $\overline{I S_{i}}=I S_{i}$. Therefore by [G, Theorem (3.1)] $S_{i}$ is a regular local ring and $I S_{i}$ is normal. Thus $S$ is regular and $I S$ is normal, whence $S=\bar{R}$.

Corollary 3.2. Let $R$ be a two-dimensional analytically unramified unmixed local ring with maximal ideal $\mathfrak{m}$ and let $I$ be an $\mathfrak{m}$-primary ideal in $R$. If $\mathrm{e}_{I}^{-1}(R)=0$, then $\bar{R}$ is a regular ring and $I \bar{R}$ is normal.

Proof. Notice that $\bar{R}$ is a finitely generated $R$-module and $\operatorname{depth}_{R} \bar{R}=2$, because $R$ is analytically unramified and unmixed with $\operatorname{dim} R=2$, whence the assertion follows from Theorem 3.1 taking $S=\bar{R}$.

Remark 3.3. The ring $R$ itself is, however, not necessarily a regular local ring even if $\operatorname{dim} R=2$. Let us note an example. We look at the local ring

$$
R=k[[X, Y, Z, W]] /[(X, Y) \cap(Z, W)],
$$

where $k[[X, Y, Z, W]]$ is the formal power series ring over a field $k$. We then have $\overline{\mathrm{e}}_{\mathfrak{m}}^{1}(R)=0$ and $\overline{\mathrm{e}}_{\mathfrak{m}}^{2}(R)=-1$. The ring $R$ is Buchsbaum but not Cohen-Macaulay, while

$$
\bar{R}=k[[X, Y]] \times k[[Z, W]]
$$

is a regular ring. 


\section{REFERENCES}

[G] S. Goto, Integral closedness of complete-intersection ideals, J. Algebra, 108 (1987), 151-160. MR887198 (88d:13015)

[GhGHOPV] L. Ghezzi, S. Goto, J. Hong, K. Ozeki, T. T. Phuong, and W. V. Vasconcelos, Cohen-Macaulayness versus the vanishing of the first Hilbert coefficient of parameter ideals, J. London Math. Soc., 81 (2010), 679-695.

[HU] J. Hong and B. Ulrich, Specialization and integral closure, preprint (2006).

[M] H. Matsumura, Commutative Algebra, second edition, Mathematics Lecture Note Series, The Benjamin/Cummings Publishing Company, Inc., 1980. MR575344 (82i:13003)

[MSV] M. Mandal, B. Singh, and J. Verma, On some conjectures about the Chern numbers of filtrations, arXiv:1001.2822v1 [math.AC].

[MTV] M. Moralès, N.V. Trung, and O. Villamayor, Sur la fonction de Hilbert-Samuel des clôtures intégrales des puissances d'idéaux engendrés par un système de paramètres, J. Algebra, 129 (1990), 96-102. MR:1037394(91b:13021)

[N] M. Nagata, Local Rings, Interscience, 1962. MR0155856 (27:5790)

[Nr] M. Narita, A note on the coefficients of Hilbert characteristic functions in semiregular rings, Proc. Cambridge Philos. Soc., 59 (1963), 269-275. MR0146212 (26:3734)

[NR] D. G. Northcott and D. Rees, Reductions of ideals in local rings, Proc. Camb. Phil. Soc., 50 (1954), 145-158. MR0059889(15:596a)

[PUV] C. Polini, B. Ulrich, and W. V. Vasconcelos, Normalization of ideals and BrainçonSkoda numbers, Mathematical Research Letters, 12 (2005), 827-842. MR2189243 (2006m:13006)

[V] W. V. Vasconcelos, The Chern coefficients of local rings, Michigan Math. J., 57 (2008), 725-743. MR 2492478 (2009m:13005)

Department of Mathematics, School of Science and Technology, Meiji University, 1-1-1 Higashi-Mita, TAMA-KU, KaWASAKI 214-8571, JaPAN

E-mail address: goto@math.meiji.ac.jp

Department of Mathematics, Southern Connecticut State University, 501 Crescent Street, New Haven, Connecticut 06515-1533

E-mail address: hongj2@southernct.edu

Department of Mathematics, Indian Institute of Technology Bombay, Powai, MumBAI, 400076, INDIA

E-mail address: mousumi@math.iitb.ac.in 